\title{
Vertical plane ex vivo dermoscopy: a novel dermoscopic technique. A preliminary study*
}

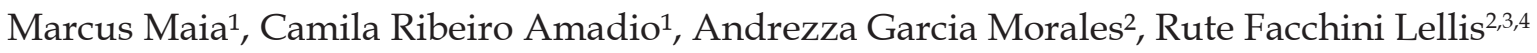

DOI: http:/ /dx.doi.org/10.1590/abd1806-4841.20187863

\begin{abstract}
Dermoscopic examination allows visualization of horizontal images on the skin, showing well-defined patterns. The authors propose ex vivo dermoscopic visualization using a vertical view of skin sections. The new image clearly distinguishes the stratum corneum, epidermis, and dermis as well as the anatomical location of the pigment. Will this new approach be useful in dermoscopic diagnosis? Further studies are needed.
\end{abstract}

Keywords: Dermoscopy; Diagnosis; Histology; Melanoma

Dermoscopy is an essential tool for diagnosis of melanocytic lesions. Its use with contact non-polarized light or polarized light allows visualization of the skin's horizontal plane. Diagnostic accuracy may increase by up to $49 \%$ if the dermoscopy is performed by an experienced professional. ${ }^{1}$ The technique thus enhances the dermatologist's ability to differentiate between benign and malignant lesions. ${ }^{2}$

In some cases, the correct diagnosis is made after a more extensive search for subtle clues in the biopsy specimen, driven by valorization of dermoscopic findings communicated to the pathologist. ${ }^{3}$ The structures seen under dermoscopy have specific correlates in histopathologic studies. Understanding these histopathologic correlates allows clinicians to better assess the dermoscopic features of pigmented lesions. ${ }^{4}$
Scope et $a .^{3}$ analyzed the role of dermoscopy in clinical practice $^{1}$ and proposed the use of ex vivo dermoscopic examination to identify areas of greatest concern and orient tissue sections by the pathologist. The methodology led to optimal correlation between clinical presentation and histopathology. This is essential, since gaps between the clinical presentation and histopathology could lead to diagnostic discordance. ${ }^{5,6}$

In a previous article, the current authors used horizontal $e x$ vivo dermoscopy to correlate clinical and histopathologic findings in lesions and demonstrated that the areas of greatest concern in dermoscopy corresponded to those with disruption of the cytoarchitecture. ${ }^{6}$ While processing sections in suspicious areas, dermoscopy was performed on the section's vertical side, allowing clear identification of the stratum corneum, epidermis, and the limit with

\footnotetext{
Received 15 November 2017.

Accepted 21 January 2018

* Work conducted at the Dermatology Clinic, Santa Casa de São Paulo, São Paulo (SP), Brazil.

Financial support: None.

Conflict of interest: None.

Dermatology Clinic, Santa Casa de São Paulo, São Paulo (SP), Brazil.

Department of Pathology, Santa Casa de São Paulo, São Paulo (SP), Brazil.

Dermatopathology Sector, Santa Casa de São Paulo, São Paulo (SP), Brazil

Dermatopathology Sector, Hospital A.C. Camargo, São Paulo (SP), Brazil.

MAILING AdDRESS:

Marcus Maia

E-mail: marcusmaiasp@uol.com.br

C2018 by Anais Brasileiros de Dermatologia
} 
the dermis, regardless of the presence or absence of pigment (chromophores), probably due to the structures' different refractive indices. Surprisingly, it was also possible to observe the dermoscopic images of the chromophores, including identification of their anatomical location.

To better demonstrate this technique of ex vivo dermoscopy applied to the vertical plane, we selected a phototype-IV patient presenting a pigmented lesion on the left plantar region, with several years' evolution (Figure 1). First, dermoscopic examination was performed, allowing visualization of the parallel ridge pattern, supporting the suspicion of an acral lentiginous melanoma (Figure 2).

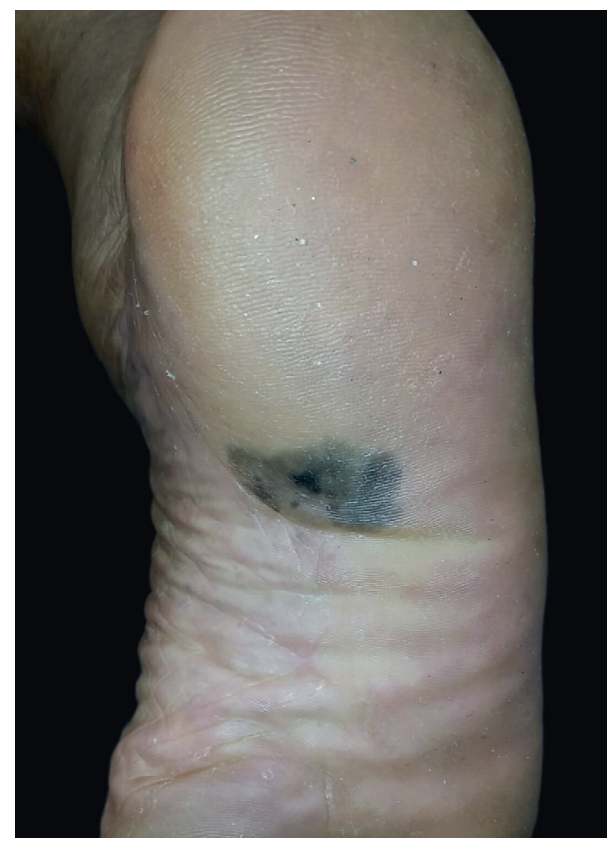

Figure 1: Dermatosis located on the plantar cavum characterized as an irregular macule with multiple shades, from black to grayish, measuring $3 \times 2 \mathrm{~cm}$

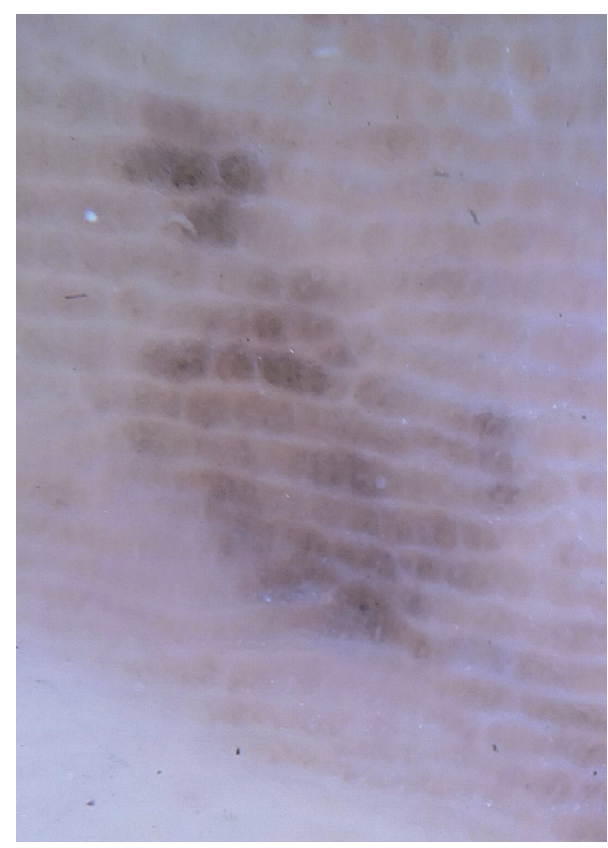

Figure 2:

Dermoscopy showing parallel and homogeneous ridge pattern in the lesion
This was followed by a biopsy, and with the aid of the dermoscope, the dermatopathologist performed a perpendicular slice to the parallel ridges (Figure 3). The aim of this plane was to better observe the anatomical characteristics of the plantar region, whose epithelial ridges are longitudinal and interspersed by the intermediate ridges, where the adnexal duct of the sweat glands is located. ${ }^{7}$ Dermoscopic examination of the vertical plane allowed visualization of the thick stratum corneum and the epidermis and its clear limit with the dermis. Based on the type of section, it was also possible to identify the border ridges, including the intermediate ridges. The location of the chromophores (melanin) was circumscribed to the intermediate ridges and often marked the intraepithelial duct of the sweat glands, reaffirming the parallel ridge pattern (Figure 4). Anatomopathological examination, usually performed on the same plane as

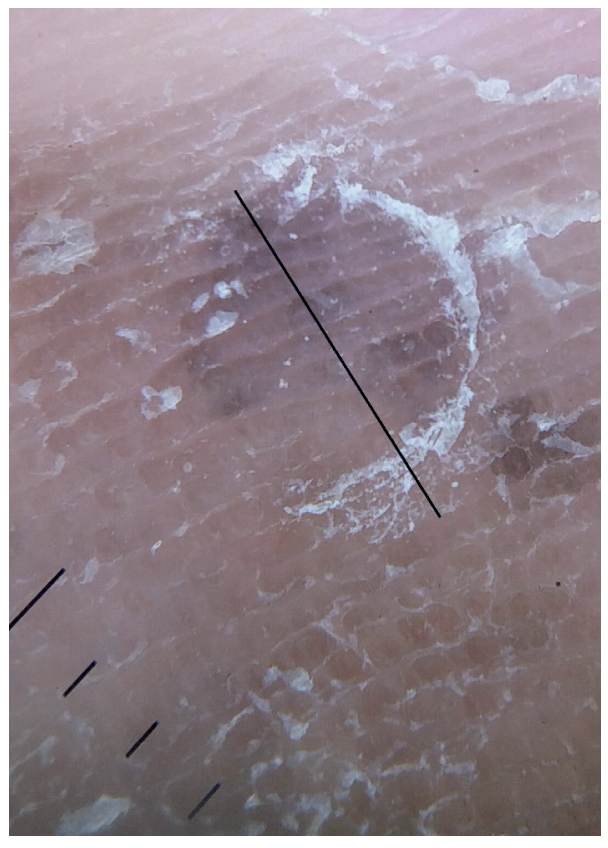

Figure 3: Punch biopsy $\left(\mathrm{n}^{\circ} .4\right)$; line represents the direction of the section in the specimen, perpendicular to the parallel ridges

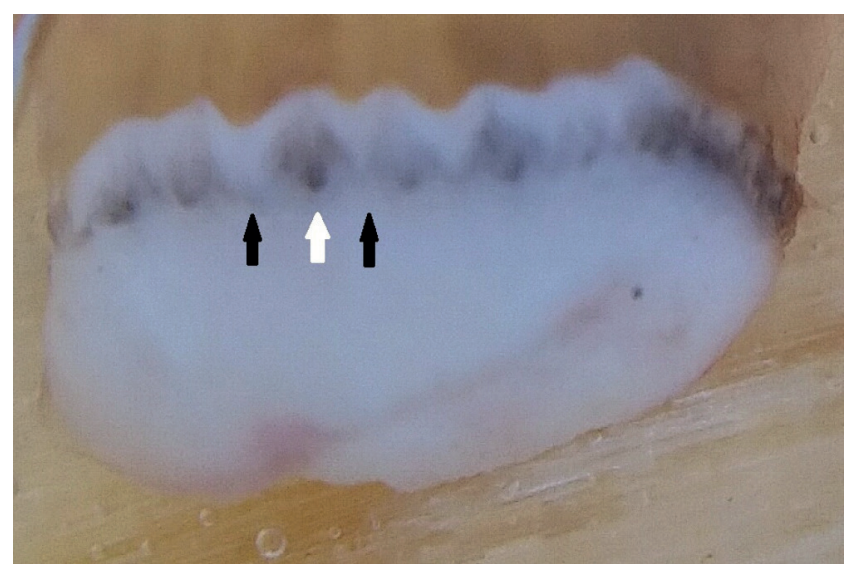

FIGURE 4: Melanin limited to the intermediate ridges and also marking the intraepithelial duct of the sweat glands. White arrow shows the intermediate ridge. The black arrow showing the limiting ridges 


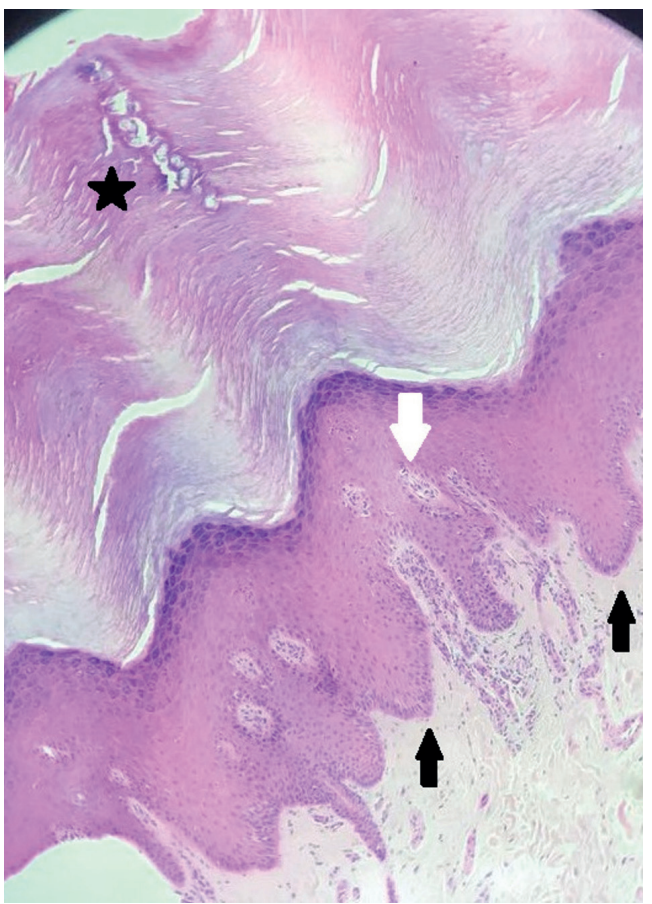

FigURE 5: Histologic section of plantar skin shows hyperparakeratosis with melanocytic atypia in the basal layer of epidermal ridges with pleomorphism and oriented upwards, characterizing malignant melanocytic lesion/acral melanoma. White arrow shows the intermediate ridge. Black arrow shows the limiting ridges. The star represents the glandular duct this dermoscopic method, showed perfect correlation between the vertical dermoscopic image and histopathology (Figure 5). Anatomical pathology revealed acral melanoma. The areas of greatest concern in dermoscopy matched those with the greatest cytoarchitectural disruption. Ex vivo examination allowed determining the exact sites for sections and correlation with pathology.

This dermoscopic technique applied to a case of acral lentiginous melanoma led to more accurate diagnosis, suggesting its potential usefulness for other diagnoses such as melanocytic nevi.

The longitudinal biopsy section combined with dermoscopy improved the correlations between the in vivo lesion and the ex vivo dermoscopy for both clinicians and pathologists, thus aiding identification of the lesions' typical features. Vertical plane ex vivo dermoscopy is intended to better correlate the lesions' clinical information with their distinctive histopathologic features in order to optimize diagnosis and reduce possible misunderstandings that may occur in the diagnosis of benign versus malignant melanocytic lesions, potentially with better outcomes in both situations.

In summary, vertical plane ex vivo dermoscopy supported the suspicion of clinical and horizontal dermoscopic features of a lesion through the precise anatomical localization of the pigmentary process and its anatomopathological correlation. New observations are needed to assess whether this new approach will add value to the diagnosis and characterize new dermoscopic patterns on the skin's vertical plane. $\square$

\section{REFERENCES}

1. Kittler H, Pehamberger H, Wolff K, Binder M. Diagnostic accuracy of dermoscopy. Lancet Oncol. 2002;3:159-65.

2. Braun RP, Thomas L, Dusza SW, Gaide 0, Menzies S, Dalle S, et al. Dermoscopy of acral melanoma: a multicenter study on behalf of the International Society of Dermoscopy. Dermatology. 2013;227:373-80.

3. Scope A, Busam KJ, Malvehy J, Puig S, McClain SA, Braun RP, et al. Ex vivo dermoscopy of melanocytic tumors: time for dermatopathologists to learn dermoscopy. Arch Dermatol. 2007;143:1548-52.

4. Braun RP, Kaya G, Masouyé I, Krischer J, Saurat JH. Histopathologic correlation in dermoscopy: a micropunch technique. Arch Dermatol. 2003;139:349-51.
5. Miyazaki A, Saida T, Koga H, Oguchi S, Suzuki T, Tsuchida T. Anatomical and histopathological correlates of the dermoscopic patterns seen in melanocytic nevi on the sole: a retrospective study. J Am Acad Dermatol. 2005;53:230-6.

6. Maia M, Lellis RF, Marta AC. Dermatoscopia ex vivo: avaliação sincrônica entre 0 dermatologista e 0 dermatopatologista de lesões melanocíticas - Estudo prévio. An Bras Dermatol. 2009;84:553-55.

7. Saida T, Oguchi S, Miyazaki A. Dermoscopy for acral pigmented skin lesions. Clin Dermatol. 2002;20:279-85.

Statistical analysis, Approval of the final version of the manuscript, Conception and planning of the study, Elaboration and writing of the manuscript, Obtaining, analyzing and interpreting the data, Effective participation in research orientation, Intellectual participation in propaedeutic and/or therapeutic conduct of the cases studied, Critical review of the literature, Critical review of the manuscript

Camila Ribeiro Amadio

iD ORCID 0000-0001-8980-9815

Statistical analysis, Approval of the final version of the manuscript, Conception and planning of the study, Elaboration and writing of the manuscript, Obtaining, analyzing and interpreting the data, Intellectual participation in propaedeutic and/or therapeutic conduct of the cases studied, Critical review of the literature, Critical review of the manuscript Andrezza Garcia Morales $\quad$ (iD) ORCID 0000-0003-0560-6047

Statistical analysis, Approval of the final version of the manuscript, Conception and planning of the study, Elaboration and writing of the manuscript, Obtaining, analyzing and interpreting the data, Intellectual participation in propaedeutic and/or therapeutic conduct of the cases studied, Critical review of the literature, Critical review of the manuscript

Rute Facchini Lellis

(iD) ORCID 0000-0001-7690-0513

Statistical analysis, Approval of the final version of the manuscript, Conception and planning of the study, Elaboration and writing of the manuscript, Obtaining, analyzing and interpreting the data, Effective participation in research orientation, Intellectual participation in propaedeutic and/or therapeutic conduct of the cases studied, Critical review of the literature, Critical review of the manuscript

How to cite this article: Maia M, Amadio CR, Morales AG, Lellis RF. Vertical plane ex vivo dermoscopy: a novel dermoscopic technique. A preliminary study. An Bras Dermatol. 2018;93(6):899-901. 